\title{
Distal Tumors Elicit Distinctive Gene Expression Changes in Mouse Brain, Different from Those Induced by Arthritis
}

\author{
Mariano J. Alvarez ${ }^{\#, 1}$, Mariano C. Salibe $\#$, , Gustavo Stolovitzky², Marcelo Rubinstein ${ }^{3}$, Fernando J. \\ Pitossi*,4 and Osvaldo L. Podhajcer*,4 $^{*}$ \\ ${ }^{1}$ Gentron Research Unit, Roque Sáenz Peña 778 (1642) Buenos Aires, Argentina \\ ${ }^{2}$ Functional Genomics \& Systems Biology, IBM T.J. Watson Research Center, New York, USA \\ ${ }^{3}$ INGEBI, CONICET, Buenos Aires, Argentina \\ ${ }^{4}$ Laboratory of Molecular and Cellular Therapy, Instituto Leloir, CONICET, Buenos Aires, Argentina
}

\begin{abstract}
Background: Tumor progression is characterized by high mutation rates, each mutation potentially generating an "alarm" signal. The brain is the main integrator of signals arising in the periphery from changes in homeostasis. We hypothesized that tumors growing at a distant site might be a stimulus strong enough to be molecularly sensed and integrated by the brain.

Results: Transcriptome analysis of the mouse hypothalamus, midbrain, and pre-fontal cortex at different time points following administration at a distant site of mammary, lung and colon cancer cells evidenced cancer-type and brain-region specific changes in gene expression. On the contrary, no significant gene expression changes were detected in the liver. The hypothalamus was the region with the largest number of differentially expressed genes. On the array and off the array analysis of hypothalamic samples using real time PCR confirmed changes in genes associated with synaptic activity and sickness response, respectively. Gene clustering allowed the discrimination between each cancer model and between the cancer models and arthritis.
\end{abstract}

Conclusions: The present data provides evidence of changes in gene expression in the brain during progression of distal tumors and arthritis highlighting a potential link between distal pathological processes and the brain.

\section{INTRODUCTION}

Tumor progression is characterized by high genetic instability, each mutation producing a new genetic clone that will potentially promote an "alarm" signal inducing the recruitment and activation of immune responses [1, 2]. In a simple view, thousands of "alarm" signals might be produced during the time course of tumor development before it is detected in the clinics [2]. The nervous system maintains body homeostasis by sensing and reacting to signals that reach a certain threshold [3-7]. Maintenance of homeostasis not only involves adaptations to changes due to normal organ function but also during disease states. The brain can sense immune peripheral events through soluble compounds or the vagus nerve, and can react for example through activation of the hypothalamus-pituitary-adrenal axis, resulting in the modulation of an ongoing immune response $[8,9]$. In addition, the autonomic nervous system innervates lymphoid tissues hardwiring the brain and peripheral organs involved in immune reactions such as the spleen and the thymus, providing the possibility of an immediate sensing by the brain of events related to peripheral diseases $[6,10]$.

*Address correspondence to these authors at the Laboratory of Molecular and Cellular Therapy, Instituto Leloir, CONICET, Buenos Aires, Argentina; Tel: (54-11) 4747-4848; Fax: (54-11) 4742-2571;

E-mail: fpitossi@leloir.org.ar; opodhajcer@leloir.org.ar

"These authors contributed equally to this work.
Few studies in animal models suggested the potential involvement of the central nervous system (CNS) in tumor growth. For instance, injection of tumorigenic cells has been shown to activate neuroendocrinal axes [4], and mice bearing tumors showed enhanced norepinephrine and serotonin catabolism and increased tryptophan levels in the brain [11]. These few studies suggesting a potential link between tumor development and the CNS led us to hypothesize that the presence of "foreign" tumorigenic cells at a distal site could be a stimulus strong enough to be molecularly "sensed" by the brain, long before growing tumors become noticeable by conventional methods. We report here a comprehensive microarray analysis to address whether specific changes in gene expression are triggered predominantly in discrete brain regions in the presence of a tumor growing at a distal site. The present data shows changes in the expression levels of a set of genes in the brain as early as $18 \mathrm{~h}$ after administration of different types of tumor cells. Only a low percentage of differentially expressed genes were shared among the different tumor models. The hypothalamus showed the largest amount of differentially expressed genes. On the array and off the array analysis highlighted genes involved in neuronal connectivity and sickness behavior, respectively. Gene clustering and leave-one-out cross-validation showed that the different tumors can be discriminated using the brain gene expression profile. Moreover, clustering of brain expressed genes also segregated all arthritic samples from each of the three tumor models with perfect accuracy. 


\section{MATERIALS AND METHODOLOGY}

\section{In Vivo Studies}

CT-26.WT colon cancer, 4T-1 mammary cancer, and LL/2(LLC1) lung cancer cells were obtained from the American Type Culture Collection (ATCC, Manassas, VA). Eight week old male and female Balb/c and male C57Bl/6 mice housed in HEPA filtered air racks (Tecniplast, Italy) with food and water ad libitum were injected s.c. with $1 \times 10^{6}$ CT-26.WT, $1 \times 10^{5} 4 \mathrm{~T}-1$ or $1 \times 10^{6}$ LL/2(LLC1) cells in PBS; control mice were injected with PBS alone. Mice were killed by cervical dislocation and brains were dissected; in some experiments, also the liver was extracted. All tissue samples were frozen in dry ice and stored at $-80^{\circ} \mathrm{C}$ until RNA extraction.

\section{Burrowing}

This test was essentially performed as described [12]. Briefly, two hours before the start of the dark period, mice were placed in individual plastic cages containing plastic tubes (20 cm long, $4 \mathrm{~cm}$ diameter) filled with pellets. The lower closed end was resting on the cage floor. The open end was supported $3 \mathrm{~cm}$ above the cage floor with screws, preventing the contents from being non-purposefully displaced. The tube was filled with $300 \mathrm{~g}$ of pellets. The weight of pellets remaining in the tube was measured $16 \mathrm{~h}$ later, from which the weight displaced (burrowed) was calculated. As positive control for sickness behavior symptoms, similar experiments were done with mice injected with LPS at a 2 $\mathrm{mg} / \mathrm{kg}$ dose. These mice showed no borrowing activity.

\section{Forced Swimming Test}

Mice were individually placed in cylinders $(19 \mathrm{~cm}$ in diameter and $25 \mathrm{~cm}$ high) filled with water $\left(24 \pm 1{ }^{\circ} \mathrm{C}\right)$ up to $15 \mathrm{~cm}$ deep. Time to immobility was recorded [13]. Immobility was considered when mice only did minimal movements to maintain floatability. After the end of the experiment, mice were dried with a towel and placed back in the cage.

\section{Microarray Studies}

Total RNA was extracted using TRIzol Reagent (Invitrogen, Carlsbad, CA). Poly A+ RNA was obtained from total RNA using the MicroPoly(A) Pure kit (Ambion, Austin, Texas). RNA was reversed transcribed using Superscript II RT (Invitrogen) with oligo dT primers and random primers, both in the presence of aa-dUTP (Sigma Co., St Louis, MO). The cDNA was labeled with Cy3 or Cy5 dyes (Amersham Pharmacia, Sweden), resuspended in DMSO, and incubated for $1 \mathrm{~h}$ at room temperature in the dark. The probes were purified using the SNAP Gel Purification Kit (Invitrogen) following manufacturer instructions with the following modification: at the initial step, $500 \mu \mathrm{l}$ of loading buffer (2.25 $\mathrm{M}$ guanidinium $\mathrm{HCl}$ in 70\% isopropanol) were added to the sample, placed in a SNAP column and incubated for 4 min before the first centrifugation. 50-mer mouse 10K Oligo Set (MWG, Germany) were printed on UltraGAPS slides (Corning, Acton, MA) using a Virtek Chipwriter Arrayer (Virtek Vision International Inc. Ontario, Canada). Printed slides were prehybridised at $42^{\circ} \mathrm{C}$ in $2 \mathrm{X}$ SSC, $0.1 \%$ SDS, $1 \%$ BSA. Labeled probes were mixed with hybridization buffer containing $30 \%$ formamide and hybridized overnight at $42^{\circ} \mathrm{C}$, using a dye swap design.

\section{Real-Time PCR}

$0.5 \mu \mathrm{g}$ of mRNA was reverse-transcribed using oligo(dT) ${ }_{12-18}$ (Invitrogen) and Superscript II RNaseH' Reverse Transcriptase (Invitrogen) following manufacturer instructions. Quantification of cDNA was performed using Oligreen ssDNA Quantitation Reagent (Invitrogen). Primers were designed using the Primer3 program (http:// www-genome.wi.mit.edu/cgi-bin/primer/primer3.cgi/primer3 www.cgi). Each gene was analyzed by comparing with two housekeeping genes (beta2-microglobulin and beta-actin) in 96-well optical plates on an iCycler IQ Real-Time Detection System (Bio-Rad) using SYBR Green I (Invitrogen). Melting curves of all samples were always performed. Each target gene was assessed $2-4$ times, each time in triplicates. The initial template amount in fluorescent units $\left(T_{0}\right)$, was estimated as described in [14]. Briefly, $T_{0}$ was calculated as $T_{0}=$ $T_{C T}(1+E)^{-C T}$, where $T_{C T}$ is the amount of product (fluorescent units) at the threshold cycle $C T$. The intrinsic amplification efficiency $(E)$ was calculated as

$$
E=\left(1+e^{-\left(\frac{T m}{b}\right)}\right)^{-1}
$$

and the parameters $T m$ and $b$ were estimated by fitting the amplification data to the function

$\frac{T_{n+1}}{T_{n}}=1+\left(1+e^{\left(\frac{T_{n}-T m}{b}\right)}\right)^{-1}$

by non-lineal regression.

Differential expression was assessed by ANOVA of 4 to 6 experiments.

\section{Data Analysis}

All the data processing was performed with the R System v2.2.1 (The R Project for Statistical Computing).

\section{Data Acquisition and Image Processing}

The slides were scanned with a Chip-Reader (Bio-Rad, Hercules, CA) at $10 \mu \mathrm{m}$ resolution and 16 bit pixel depth, and images were analyzed with VersArray Analyzer software v4.5 (BioRad).

\section{Data Filtration and Normalization}

The data was filtered to eliminate dust derived data points (spots with size less than 75 pixels or with a mean to median correlation of less than $80 \%$ [15], saturated data points (spots with a proportion of saturated pixels greater than 20\%), and low signal data points (spots with signal to background ratio below 1.2).

Data was quality-filtered, and slides were normalized using an intensity- and location-dependent algorithm. Briefly, the intensity ratio for spot $i\left(M_{i}=\log _{2} R_{i} / G_{i}\right)$ was replaced by the residuals of a locally-weighted 3Dpolynomial surface regression $M_{i}=f\left(x_{i}, y_{i}, A_{i}\right)$, where $\left(x_{i}, y_{i}\right)$ is the location of spot $i$ in the slide and $A_{i}$ is the mean spot intensity; i.e. $A_{i}=1 / 2 \cdot \log _{2} R_{i} / G_{i}$. The 3D-normalization 
scheme was applied sequentially in two steps, first on the entire slide to eliminate bias that span across grids, and then on each grid separately, to eliminate print-tip dependent bias. Locally weighted 3D polynomial regression was carried out with the loess function of the R-system. $M$ values from dyeswap (technical) replicates were weighted by their quality scores (QC) and averaged. QC was defined as the ratio of spot area to spot perimeter scaled in a range $(0,1]$. The resulting expression set was scale-normalized so that each sample has the same median absolute deviation [16].

\section{Analysis of Biological Replicates}

We employed a mixed effects experimental design with factor treatment (levels: tumor and control) and random effect experiment (levels: 1-4) without replication. The statistical significance for the treatment factor was estimated by ANOVA [17]. For the identification of genes affected by the factor treatment at either time point, we added the factor time (levels, 18, 72 and $192 \mathrm{~h}$ ) to the previous design instead of performing multiple ANOVA tests, because the former had greater sensitivity without loss of selectivity when compared with multiple one-way ANOVA or t-tests [18]. To identify genes for which expression levels were increased or decreased throughout the experiment, we estimated the statistical significance for the average difference between treatments by ANOVA. Genes showing a p-value $<0.05$ and an expression change higher than $20 \%$ at least in one time point were considered "differentially expressed genes".

For the identification of early and late response genes, we performed a template matching analysis using the templates $\{2,1,0\}$ and $\{0,1,2\}$, respectively [17].

\section{Functional Analysis}

Biological Process categories of the Gene Ontology database [19] enriched in differentially expressed genes were identified by Fisher's exact test. Only categories with 4 or more genes presents in our microarray platform were included in the analysis. Genes showing a fold change greater than $20 \%(\mathrm{p}<0.05)$ in any time point were considered as differentially expressed.

\section{Hierarchical Cluster Analysis}

From the list of differentially expressed genes we selected those showing differential responses between cancer models ( $\mathrm{p}<0.01$, t-test). Only experimental samples with fewer than $40 \%$ missing values and genes with fewer than $20 \%$ of missing values were included. For the Euclidean distance calculation, data was scaled as $M s_{i}=\left(M_{i}-\bar{M}\right)$ / $\mathrm{SD}(M)$, where $\mathrm{SD}$ is the standard deviation. Cluster analysis was performed using a Ward's minimum variance agglomeration method [20].

\section{Predictive Ability Estimation}

Leave-one-out cross validation was performed using a weighted-nearest neighbor classifier (see below), and the area under the Receiver Operating Characteristic curve (ROC-score) was used to determine the separating ability of the classifier. The statistical significance of the ROC-score was estimated from a null model generated by 10,000 random permutations of the class labels.

\section{Leave-One-Out Cross-Validation and ROC-Score Analysis}

If there are $n$ samples, for every sample in turn, we trained the classifier on the remaining $n-1$ samples and then we tested the resulting hypothesis on the sample left out. Classification was accomplished with a weighted version of the classical k-nearest neighbour method. Variables (genes) useful for classification were first selected from the training set (see below). The training set $(Y)$ and the test set $(X)$ were then standardized by dividing the variables by the standard deviation of $Y$. Next, the Euclidean distance $d(y, x)$ between each of the samples $y$ of the training set belonging to class $i$, and the test sample $x$ were calculated. We converted distances to weights $(w)$ by the Gauss kernel function $s^{-1}(2 \pi)^{-1 / 2}$ $\exp \left(-d^{2} /\left(2 s^{2}\right)\right)$, where $s$ is the standard deviation of the distances $d$. The decision function $f(x)$ was build using all the samples for training the $w$-nn classifier, so $k=M$. The hypothesis output value was generated as the estimated votingscore of $x$ belonging to class $0: f_{0}(x)=m_{0}{ }^{-1} \Sigma\left[w_{i}(l(i)=0)\right] / \Sigma$ $w$, where $l$ is the vector of class labels and $m_{0}$ is the amount of samples belonging to class 0 . Thus, the voting-score for the classes are:

$$
\begin{aligned}
& f_{0}(x)=\frac{1}{m_{0}} \sum_{j} \frac{1}{s \sqrt{2 \pi}} \exp \left\{-\frac{\left[d\left(x, y_{j}\right)\right]^{2}}{2 s^{2}}\right\}, \forall j \mid l_{j}=0 \\
& f_{1}(x)=\frac{1}{m_{1}} \sum_{j} \frac{1}{s \sqrt{2 \pi}} \exp \left\{-\frac{\left[d\left(x, y_{j}\right)\right]^{2}}{2 s^{2}}\right\}, \forall j \mid l_{j}=1 \\
& s^{2}=\frac{1}{M(M-1)} \sum_{i, j=1}^{M}\left[d\left(y_{j}, y_{i}\right)\right]^{2}
\end{aligned}
$$

where $m_{0}$ and $m_{1}$ are the amount of samples belonging to class 0 and 1 respectively, and $M=m_{0}+m_{1}$.

We defined the decision function as $D(x)=f_{0}(x) /\left(f_{0}(x)+\right.$ $\left.f_{1}(x)\right)$.

The LOO process produced $n$ hypothesis output values which were compared with a given detection threshold $\tau$ to predict the class. By varying $\tau$ we can draw a curve of sensitivity vs. 1-specificity of the classification, called Receiver Operating Characteristic (ROC) curve. The area under the ROC curve (ROC-score) is a measure of the separating ability of the classifier. Very good classifiers have ROC-scores close to 1; bad classifiers have ROC-scores around 0.5 . The statistical significance of the ROC-score was estimated from a null model generated by 10,000 permutations at random of the class labels. The p-value of an observed ROC-score was computed as the fraction of randomized ROC scores greater than the observed score.

To select variables (genes) suitable for classification, we searched the training set for a set of genes that maximizes the ROC-score in a LOO process. In brief, genes were rankordered according to their differential expression between classes using a t-test. The number of genes in the classifier was optimized by sequentially adding genes from the top of this rank-ordered list, starting at 2 genes and up to 100 genes. The power for correct classification was estimated for each set of genes by the ROC-score calculated with a LOO performed on the training set. The minimum amount of 
genes maximizing the ROC-score was selected as useful for classification.

\section{RESULTS}

Changes in gene expression in discrete brain regions following administration of cancer cells at a distant site

We designed an experimental protocol encompassing three cancer cell types, three brain regions and three time points of analysis. We injected Lewis lung, CT-26 colon and 4T1 mammary cancer cells into syngeneic mice and evaluated the transcriptome in the hypothalamic, prefrontal cortex and midbrain regions. We selected the hypothalamus and prefrontal cortex since in preliminary experiments we observed in these areas the highest changes in neurotransmitters levels (Casalá et al. manuscript in preparation). Midbrain was selected due to its role in the regulation of basic parameters of internal homeostasis. The injected inocula led to tumor growth in $100 \%$ of mice, a prerequisite for the further studies (see below). In order to avoid the bias and potential loss of low abundant transcripts due to RNA amplification [21], and to control for technical variability, each of the biological replicates was obtained from a pool of 15 mice.

After careful evaluation we decided to inject control mice with vehicle alone. Injection of control animals with normal cells or heat inactivated/irradiated tumor cells would eventually served as a control of non-specific responses due to the sole event of cell administration. However, normal cells will eventually die when injected in an inappropriate site sending signals of death by anoikis, which in turn could lead to a large amount of false positives/negatives results. We also ruled out the use of irradiated tumor cells since these cells do not divide, but keep secreting soluble factors that can impact the brain leading again to false positive/negative results. A similar concern was raised by the use of tumor cells supernatant. As an example of potential false positive/negative results is the fact that heat inactivated tumor cells are administered to improve the immune response generated by tumor vaccines [22].

The main concern of using sham mice as a control is that the entrance of foreign cells to an organism is not controlled. But this concern was ruled out by our experimental design that allowed each cancer model to become the control of each other in terms of gene expression induced in the different brain regions. Therefore, if changes in CNS transcriptome are independent of the cell type, these changes should be similar in every tumor model. On the contrary, if changes in CNS transcriptome are cell type dependent we should be able to find tumor-type specific gene signatures that discriminate between tumor models.

Mice were sacrificed at $18 \mathrm{~h}, 3 \mathrm{~d}$ and $8 \mathrm{~d}$ after the initiation of the experiment, and the transcriptional profile of the selected brain regions was analyzed using a replicated dyeswap design on printed-in-house $10 \mathrm{~K}$ oligonucleotide-based arrays. The data was obtained from at least four biological replicates.

A total of 658 differentially expressed genes were detected in the three cancer models $(\mathrm{P}<0.05)$. Of them, 322 were observed in the Lewis lung cancer model, 255 in the 4T1 mammary cancer model and 255 in the CT-26 colon cancer model. Fig. (1) shows the fold changes as the median \pm the 5 th and the 95 th percentiles among the differentially expressed genes ( $\mathrm{p}<0.05$ and fold change $>20 \%$ ). $22 \%$ of the total list of differentially expressed genes showed more than $50 \%$ change in transcript levels (Fig. 1A). These amplitudes of changes were consistent with previous studies performed with mammalian transcripts in the brain modulated by chronic events such as the gene expression profiles that differentiates sleep and wakefulness [23]. After false discovery rate correction to account for multiple hypothesis testing (Fig. 2), it was confirmed that the hypothalamus from the lung tumor model was the region/model that showed the highest amount of differentially expressed genes. In the three tumor models, the hypothalamus was the region with the highest amount of differentially expressed genes.

While $10.1 \%$ of differentially expressed genes $\left(\mathrm{P}<10^{-8}\right)$ were shared by the Lewis lung and 4T1 mammary cancer models, only $2.9 \%$ were shared by the Lewis lung and CT26 colon cancer models, and $2.6 \%$ were shared by the CT26 colon cancer and the 4T1 mammary cancer models. Accordingly, lung and mammary cancer models also showed the highest correlation in prefrontal cortex and hypothalamus areas, while the colon cancer and the lung cancer models where the least correlated ones (Kendall rank-correlation analysis, data not shown). These data indicate that the lung and mammary cancer models, which differed in both gender and mice strain, showed the largest number of common differentially expressed genes. These observations suggest that the different patterns of gene expression induced by the different models are not mice strain or gender driven, nor due to the amount of injected cells which differed in each model, but rather to the cell type, strongly supporting the experimental design.

We realized that transcripts sets changed their expression levels with a peak at the $18 \mathrm{~h}$ time point ("early response" genes) and others at the $8 \mathrm{~d}$ time point ("late response" genes). We reasoned that "early response" genes might include those that changed initially due to the presence of tumor cells with the subsequent offset by compensatory mechanisms. "Late response" genes might reflect transcripts sets of the established disease when the tumor is already noticeable. The administration of lung cancer cells was characterized by the induction of a large set of "early response" genes in the three brain regions with their subsequent offset, being the hypothalamus the region with the largest number of "early response" genes (Fig. 1B and data not shown). Also, administration of 4T1 mammary cancer cells induced a large number of "early response" genes in the hypothalamus and midbrain areas, while the cortical area exhibited an equivalent amount of "early" and "late response genes" (Fig. 1B). Finally, CT26 colon cancer cells administration induced the largest number of changes in the levels of "late response" genes (Fig. 1B). Thus, each cancer model exhibited a distinctive characteristic in terms of their capacity to elicit changes in brain transcriptome although the lung and mammary cancer models induced the largest changes in the number of "early response" genes. This data again suggest that changes in the CNS transcriptome are cell type-specific.

\section{FUNCTIONAL CATEGORIZATION OF THE DIF- FERENTIALLY EXPRESSED GENES}

We identified Gene Ontology (GO) biological process categories enriched in differentially expressed genes relative 
A

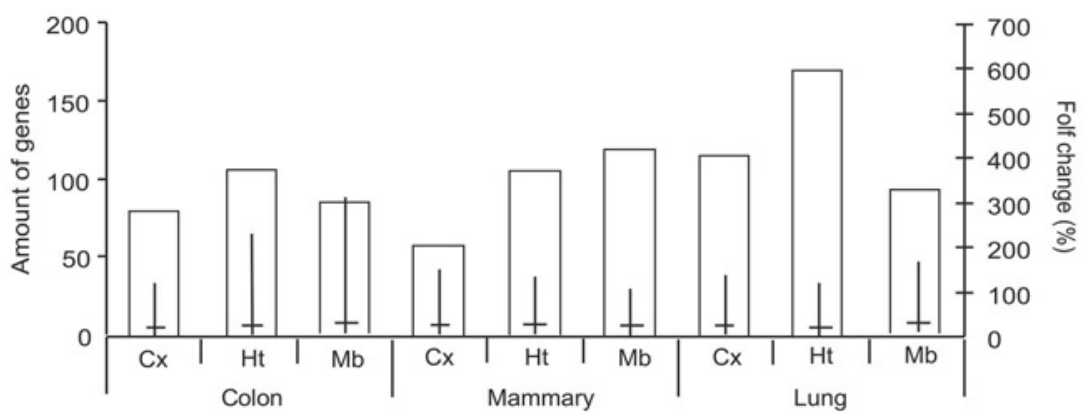

B

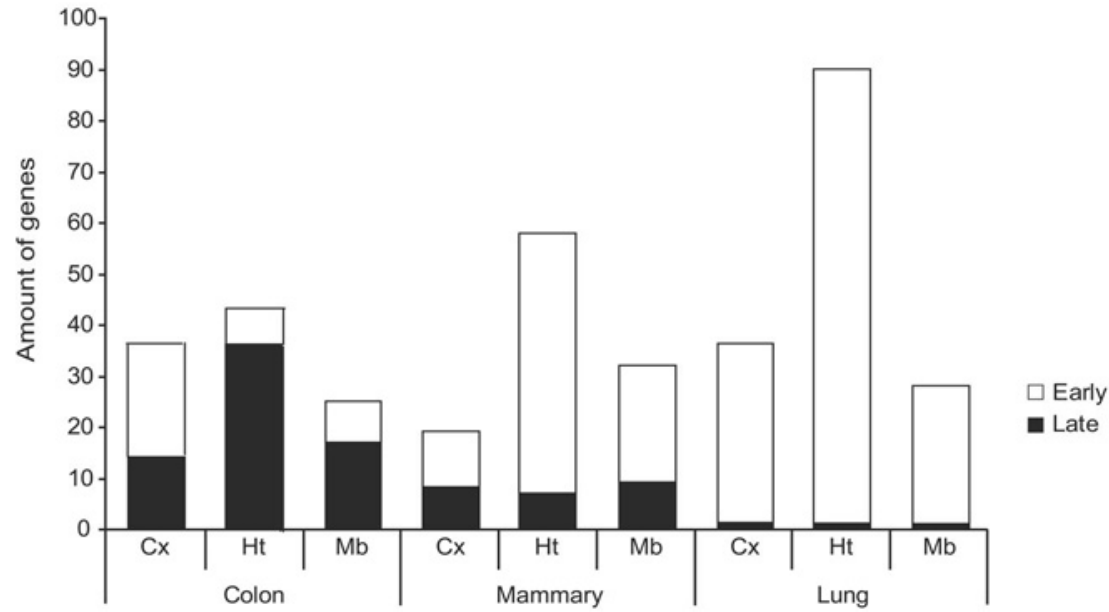

Fig. (1). Differentially expressed genes in different brain regions after injection of cancer cells at a distant site. (A) Bars are the number of differentially expressed genes ( $\mathrm{p}<0.05$ and fold change $>20 \%$ ) for each tumor model and brain area (scale in left axis). Inner horizontal and vertical lines represent the median, and the $5^{\text {th }}$ and $95^{\text {th }}$ percentiles, respectively (scale in right axis). (B) Cumulative bars represent the number of "early response" genes (white) and "late response" genes (black) for each tumor model and brain region.

Table 1. Gene Ontology Analysis of Differentially Expressed Genes. GO-Biological Processes Categories Enriched in Differentially Expressed Genes were Identified by Fisher's Exact Test. Only Significant Categories $(p<0.05)$ were Included in the Table. Categories Belonging to the Same Ancestor were Grouped and Showed in Parentheses

\begin{tabular}{|l|l|}
\hline Tumor Model & GO Categories Enriched in Differentially Expressed Genes \\
\hline \hline Lung & $\begin{array}{l}\text { biopolymer catabolism (protein catabolism), hormone secretion (peptide hormone secretion), inner ear development (inner ear morpho- } \\
\text { genesis), positive regulation of protein kinase activity, second-messenger-mediated signalling (cyclic-nucleotide-mediated signalling, } \\
\text { cAMP-mediated signalling), sensory perception (sensory perception of smell) }\end{array}$ \\
\hline Mammary & $\begin{array}{l}\text { anterior/posterior axis specification, cellular protein metabolism, DNA recombination, epithelial cell proliferation (regulation of epithe- } \\
\text { lial cell proliferation, positive regulation of epithelial cell proliferation), regulation of MAPK activity, regulation of transforming growth } \\
\text { factor beta receptor signaling pathway, sterol transport (cholesterol transport) }\end{array}$ \\
\hline Colon & $\begin{array}{l}\text { apoptotic program (apoptotic nuclear changes, disassembly of cell structures during apoptosis, DNA fragmentation during apoptosis), } \\
\text { cellular morphogenesis, cytolysis, DNA catabolism, hemostasis (blood coagulation), immune response (inflammatory response, innate } \\
\text { immune response, immune cell activation, complement activation, humoral defense mechanism, complement activation, alternative } \\
\text { pathway, complement activation, classical pathway, lymphocyte proliferation, regulation of lymphocyte activation, regulation of lym- } \\
\text { phocyte proliferation, T cell proliferation, regulation of T cell activation, regulation of T cell proliferation), regulation of cell activation, } \\
\text { response to pest pathogen or parasite }\end{array}$ \\
\hline
\end{tabular}

to the total microarray genes by performing a Fisher's exact test (cutoff $\mathrm{p}<0.05$ ). The GO data base allowed the assignment of less than $60 \%$ of the differentially expressed genes. GO analysis highlighted the peptide hormone secretion category that was enriched in the hypothalamic area of the lung cancer model (Table 1). This GO category includes two genes associated with synapsis: Pclo and Rims2 (data not shown). In addition, cAMP mediated signaling, protein catabolism, sensory perception and inner ear development were also identified, with the remaining statistically significant categories being either parents or children of these five categories in the GO hierarchy (Table 1). The hypothalamic area of the mammary cancer model that also showed a large amount of "early response" genes as the hypothalamic area 

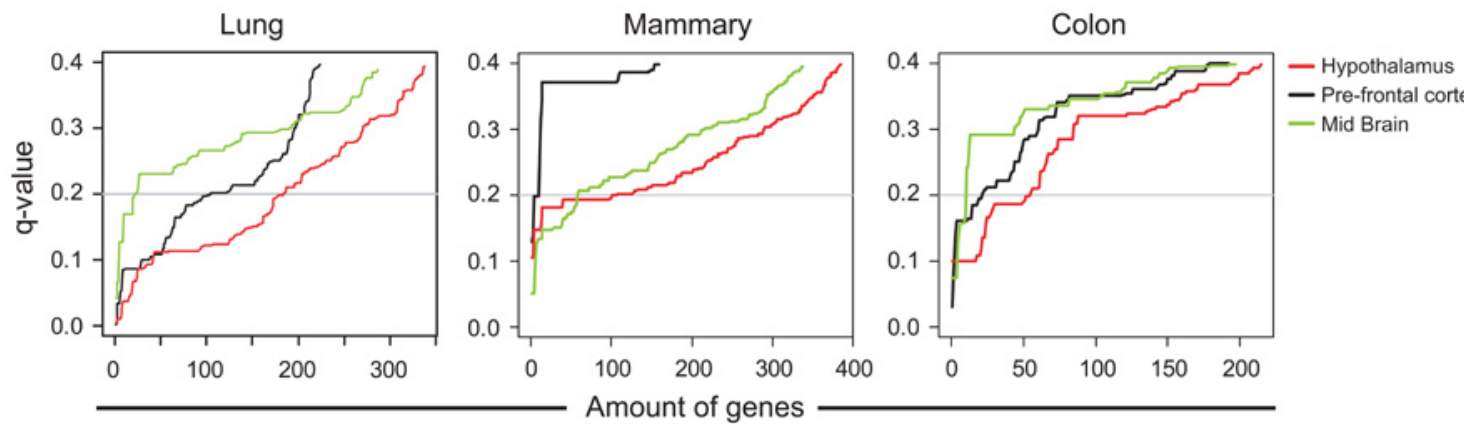

- Pre-frontal cortex

- Mid Brain

Fig. (2). False Discovery Rate (FDR; q-value) for different brain regions datasets corresponding to the three cancer models. Genes showing $>1.2$ fold change in expression levels were rank-sorted according to their p-value of differential expression. The figure shows the qvalue estimated from this ranked list.

of the lung cancer model, exhibited more general GO categories such as cellular protein metabolism, regulation of epithelial cell proliferation and DNA recombination (Table 1). Finally, the hypothalamic area of the colon cancer model that showed the highest proportion of "late response" genes mainly showed GO categories associated with immune response and apoptosis. The immune response categories included inflammatory response, complement activation, innate immune response and immune cell activation (Table 1).

\section{Real Time PCR Validation Highlights Genes Associated with Synaptic Transmission/Remodeling}

To technically validate the data we based our analysis on hypothalamic samples obtained from the Lewis lung cancer model since this region showed the largest number of differentially expressed genes and because after false discovery rate correction for multiple testing, we found a significant number of differentially expressed genes in the hypothalamic and the prefrontal region but not in the midbrain of mice (Fig. 2). Real-time PCR (qPCR) validation was performed on annotated genes selected according to their $p$ values in the microarrays, independent of the amplitude of change. Each target gene was assessed $2-4$ times, each time in triplicates. In most cases, the same gene was assessed at different time points. This selection method resulted in 55\% validation, which increased to around $80 \%$ when only samples with > 1.3 fold amplitude change in the microarrays were considered (data not shown).

Table 2 shows the list of validated genes. In some cases, only the microarray data is shown at $8 \mathrm{~d}$ to evidence the changes in gene expression levels all along the entire experiment. Few of the genes could be grouped as genes related to synaptic transmission/remodeling. That includes ataxin-2 (Atxn-2), a gene involved in dopaminergic transmission [24, 25], gamma-aminobutyric acid receptor-associated protein-like (Gabarap12) associated with GABA transmission and implicated in the fusion of synaptic vesicles, [26], and bone morphogenetic protein 15 ( $\mathrm{Bmp} 15)$, a member of the TGF-beta family involved in axon guidance [27] (Table 2). We also validated the increased expression levels of thromboxane A2 receptor (Tbxa2r) an important lipid mediator generated during oxidative stress that was associated with olygodendrocytes myelination [28], contactin-2 (Cntn2), a gene involved in remodeling of synaptic connections [29] and oxytocin (Oxt) a pleiotrophic anorexigenic molecule known as a "satiety" and "fear" hormone also involved in remodeling of synaptic connections [30] (Table 2). In addition, we also validated the down regulation of the mitochondrial ATP subunit transcript Atp5k [31], of the fibroblast activation protein, fap, an integral membrane serine protease associated with matrix remodeling [32], the DNA binding protein, kin [33] and Unc84a or SUN-1, an integral protein of the inner nuclear membrane [34]. We also validated the upregulated expression of $\mathrm{Nad}$ kinase (Nadk), the only known enzyme involved in the formation of $\mathrm{NADP}(+)$ from $\mathrm{NAD}(+)$ and ATP [35]. None of the validated genes was found to be differentially expressed in the hypothalamic areas of mice corresponding to the mammary and colon cancer models confirming that these changes are tumor type specific (data not shown). The extent of validation by real-time PCR was similar to that reported previously for low levels of differentially expressed genes in the brain [36].

\section{Expression Analysis of Genes "of the Array" Highlights Genes Associated with Sickness Behavior}

Since Tbxa2r and $O x t$ could also be assigned to an "inflammatory/immune response" category, which can trigger a "sickness behavior" state characteristic of disease progression including cancer [37], we decided to use real time-PCR to assess whether sickness behavior-associated transcripts (not present in the array) modified their expression levels. Since this process is not specific for a particular tumor type, we performed these studies in the three cancer models and brain regions.

Prostaglandin-endoperoxide synthase 2 (Cox-2) is a key enzyme in the synthesis of prostaglandin E2, a main mediator of cytokine-induced sickness behavior. We observed a slight but significant overexpression of $\operatorname{Cox}-2$ at $18 \mathrm{~h}$ and 72 $h$ in the hypothalami of mice injected with the different types of cancer cells (Table 3). Interestingly, lung and mammary, but not colon cancer cells induced also a slight but significant overexpression of Cox-2 in the prefrontal cortex at $18 \mathrm{~h}$ (Table 3). Pomc-1 levels were downregulated in the hypothalamus of mice injected with the three cancer cell types (Table 3). On the other hand, indoleamine-pyrrole 2,3 dioxygenase (Indo) levels showed immediate down regulation in the hypothalamus of the three models but returned to nonsignificantly different levels in the colon and lung cancer models at $8 \mathrm{~d}$ (Table 3). A tendency towards increased expression of arginine vasopressin ( $A v p$ ) was observed in the hypothalami of the lung and mammary cancer models (Table 3) Melanocortin receptor 4 and 5 (Mc4r and Mc5r) cortical 
Table 2. Validation of Differentially Expressed Genes by Real Time PCR

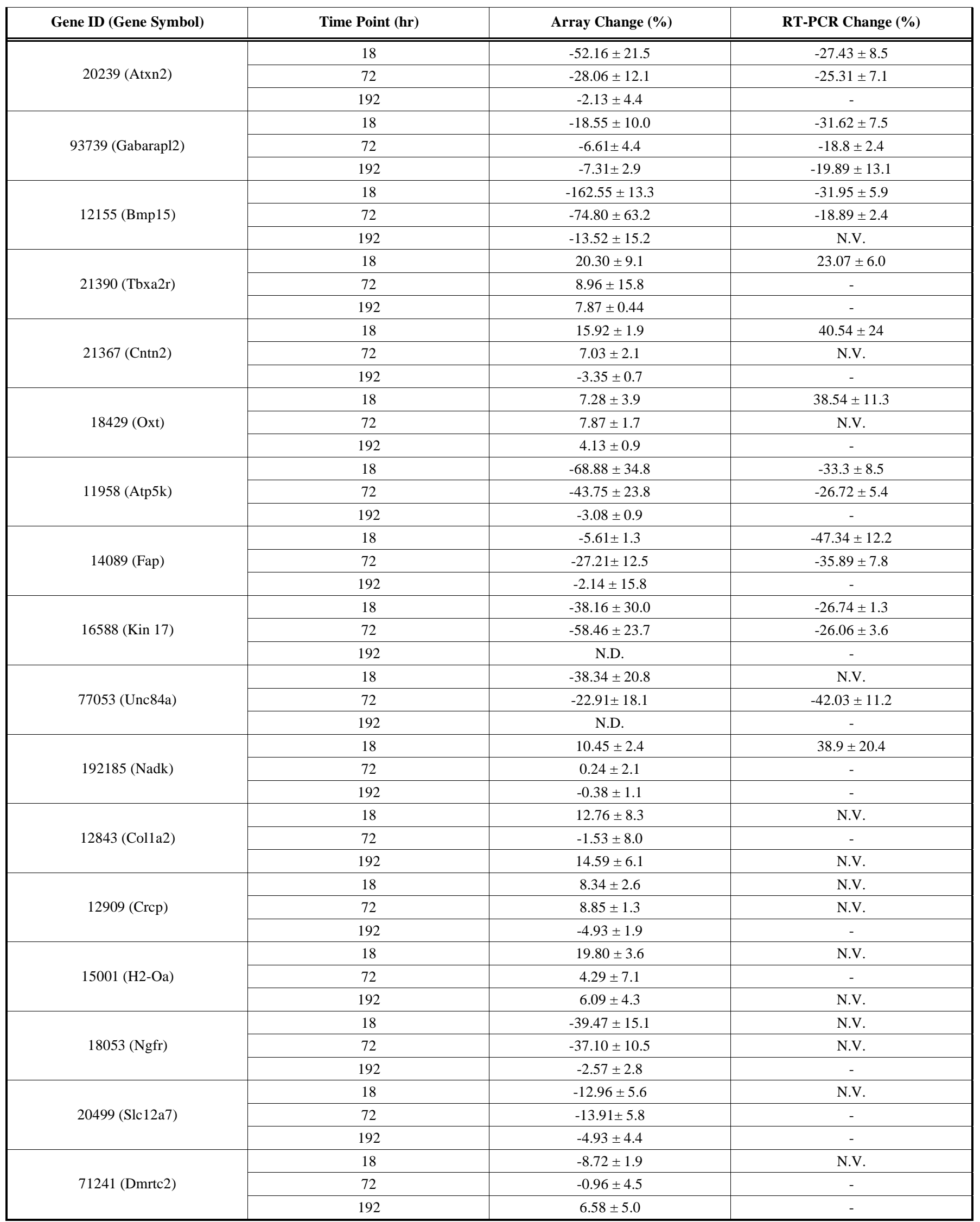


Table 3. Real-Time PCR Assessment of Sickness Behavior Related Genes

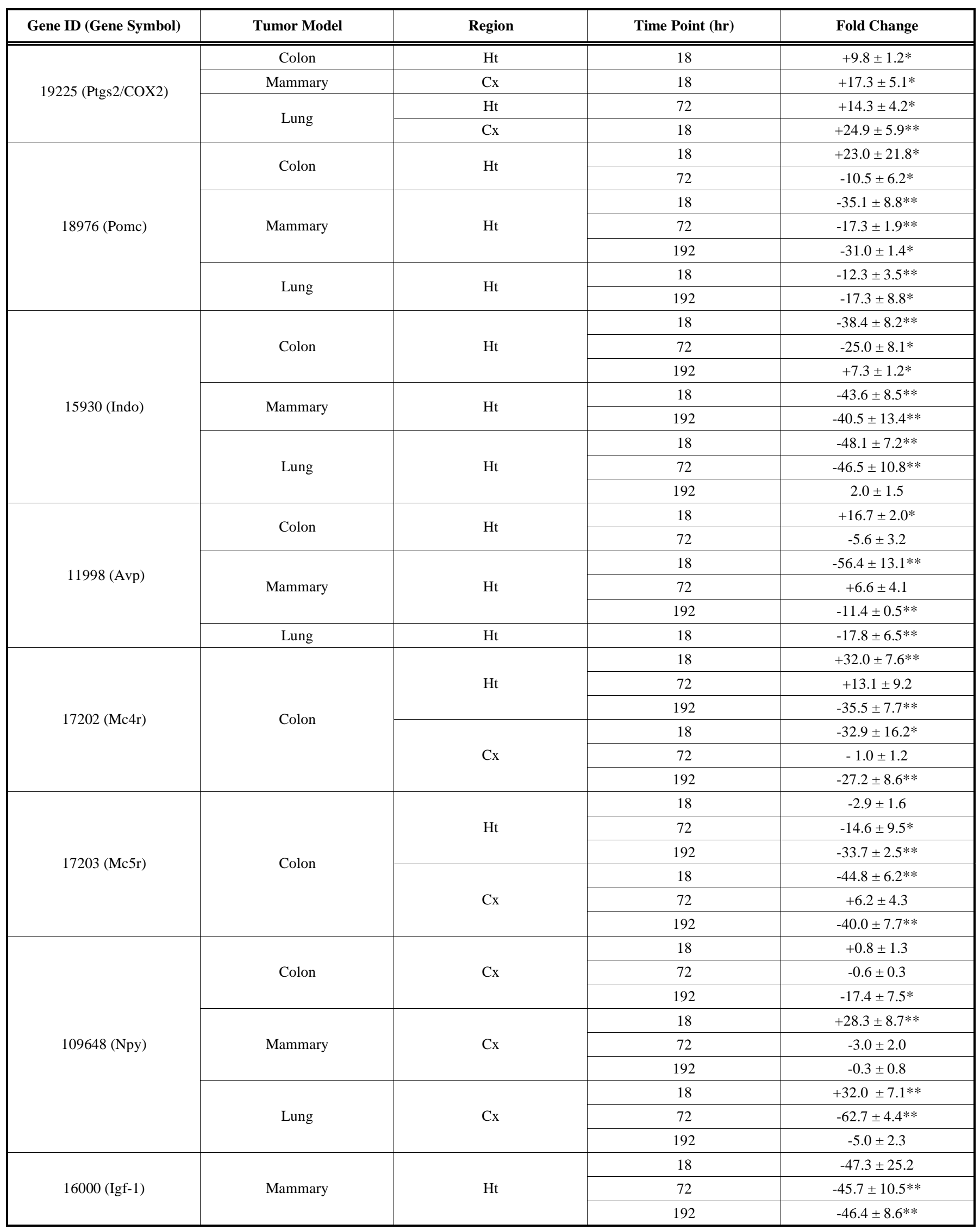


levels were essentially down regulated and mirrored one each other in the colon cancer model, while they showed an initial increase followed by a strong decrease in hypothalamic levels in the same cancer model (Table 3). Interestingly, we observed no differential expression of neuropeptide $Y$ (Npy) transcripts in the hypothalami of the three cancer models (data not shown). On the other hand, an initial increase in Npy cortical levels was observed in the mammary and lung cancer models followed by a strong decrease at $8 \mathrm{~d}$ (Table 3). In mice injected with colon cancer cells, only decreased cortical expression of Npy was observed at $8 \mathrm{~d}$ (Table 3). We observed no differentially expressed genes in the midbrain in none of the cancer models (data not shown). Despite the changes in transcript levels we observed no evidence of sickness behavior in our models as assessed by the burrowing and swimming tests [13, 38] (data not shown).

\section{No Evidence of Specific Changes in Gene Expression in the Liver}

The intimate links that communicate the brain and certain peripheral organs led us to address the question whether other organs, in addition to the brain, might also show changes in gene expression as a result of tumor cell administration. To test this possibility, mice injected with lung cancer cells or vehicle were sacrificed for evaluation of changes in transcript levels in liver using the same set of in houseprinted microarrays. Using the same cut off than in the brain studies 89 genes were identified as differentially expressed. After assessing the 11 genes showing more than $30 \%$ change by qPCR, only 1 gene could be validated ( a zinc finger gene, AN1-type domain $2 A$ (Zfand2a) with unknown function suggesting that the liver is not responding to the presence of cancer cells as the brain did.

\section{A Subset of Differentially Expressed Genes Discriminates Between the Different Tumor Types}

Our previous evidence suggested that the brain changed the expression levels of certain sets of transcripts due to the presence of a growing tumor at a distant site. Thus, we sought to establish whether a subset of differentially expressed genes would discriminate between paired cancer models. Gene clustering demonstrated that with only one exception (hypothalamic samples of the paired colon and mammary cancer models) the first branch division of the eight analyses corresponding to each brain region and paired cancer models segregated the samples corresponding to the paired cancer models with perfect accuracy, indicating that gene expression profiles in the different brain regions can discriminate between the different cancer models (Fig. 3 and Supplementary Figs. 1-3). In addition, the hypothalamic region showed statistically significant predictive ability for discriminating between lung and colon cancer (ROC-score: $0.98, \mathrm{p}<0.0001)$ and between colon and mammary cancer (ROC-score: $1, \mathrm{p}<0.0001$ ). The whole experimental data demonstrate that the changes in the CNS transcriptome are cell type-specific.

\section{A Subset of Differentially Expressed Genes Discriminates Between the Different Tumor Types and Two Arthritis Models}

To determine whether another distal chronic disease might elicit changes in brain gene expression, we assessed

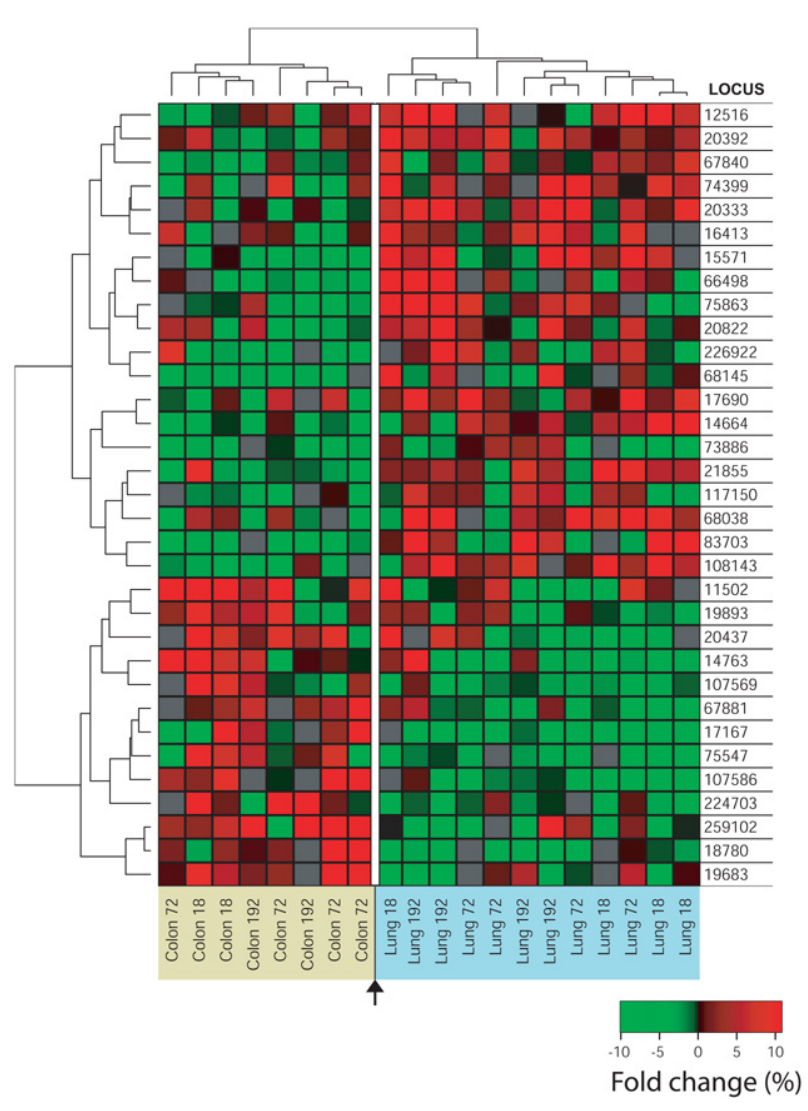

Fig. (3). Hierarchical cluster analysis of paired cancer models. Hierarchical cluster analysis of hypothalamic samples obtained from the lung and colon cancer models based on differentially expressed genes. Each column represents a sample and each row a single gene. Red indicates up-regulation, green down-regulation, and black no change. The black arrows indicate the subdivision into the dominant clusters of samples. Sample labels indicate disease model (cyan, magenta and yellow for lung, mammary and colon cancer models, respectively) and time point.

gene expression patterns in two different models of rheumatoid arthritis (RA). Microarray analysis identified 89, 73, and 104 differentially expressed genes with $>1.2$ fold change in the hypothalamus, prefrontal cortex and midbrain, respectively, compared with control mice. Data comparison demonstrated no shared genes between RA and mice injected with cancer cells (data not shown). We next sought to establish if a subset of differentially expressed genes would be able to discriminate between cancer and RA. Clustering analysis demonstrated that the first branch division segregated all RA samples from each of the three cancer models with perfect accuracy indicating that the brain can molecularly discriminate the cancer models from RA (Fig. 4 and Supplementary Figs. 4 and 5). Similarly, analysis of the 10 hypothalamic top-ranked genes in each of the 6 possible pair-wise comparisons between RA and the three cancer models showed a complete split between RA and cancer samples in the first branch strengthening the evidence that brain gene expression profiles are useful to discriminate between the two diseases (Fig. 5 and Supplementary Figs. 6 and 7). The prefrontal cortex data showed predictive ability for discrimination between RA and mammary cancer (ROC- 


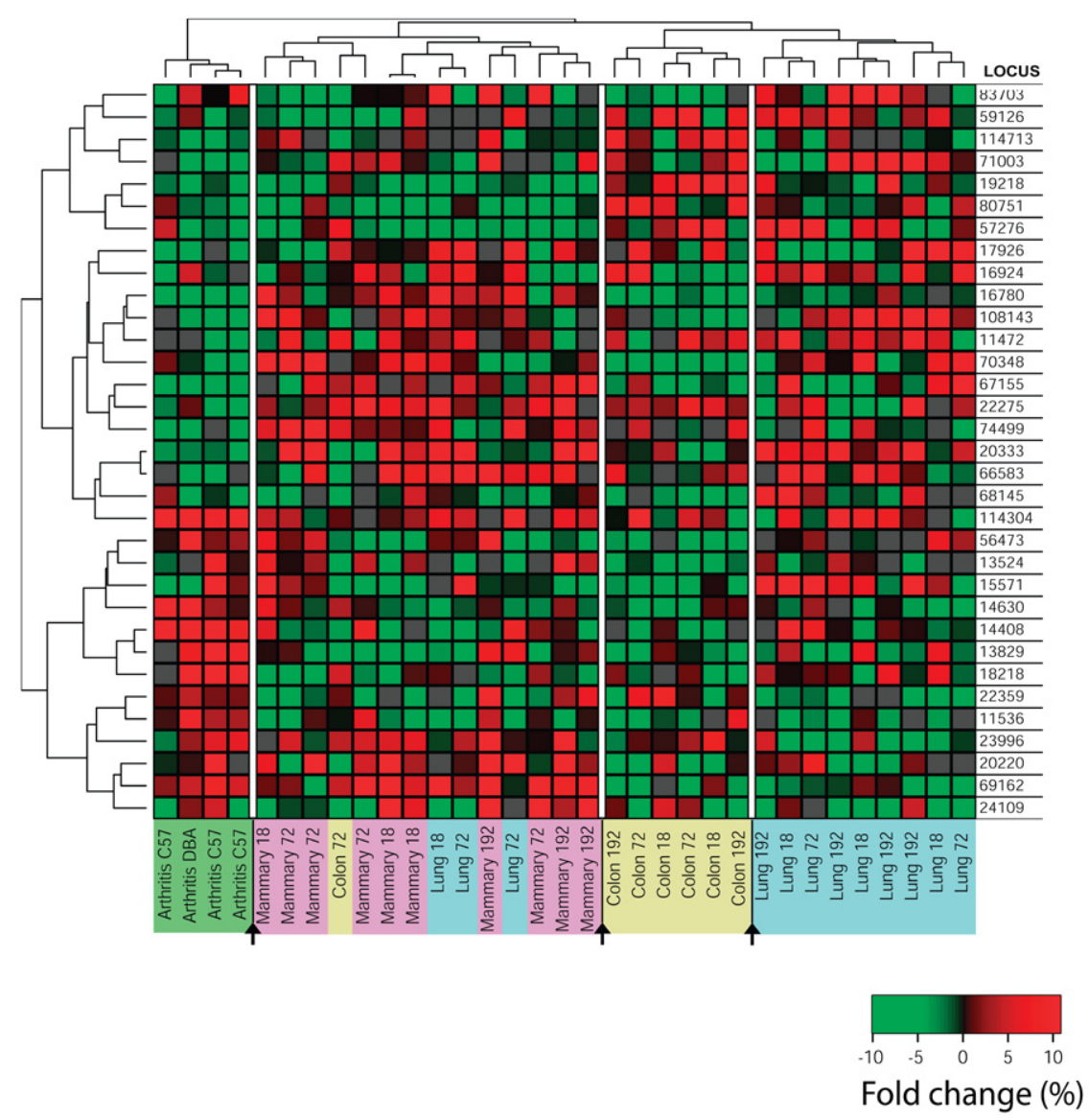

Fig. (4). Hierarchical cluster analysis of the RA and cancer models. Hierarchical cluster analysis of hypothalamic samples obtained from the arthritis and cancer models based on the 10 top-ranked genes from each pair-wise comparison. For more details see Fig. (3).

score, 0.8333; $\mathrm{p}<0.001$ ) and RA and lung cancer (ROCscore, $0.8013 ; \mathrm{p}<0.001)$, whereas the hypothalamic data could be used to discriminate between RA and colon cancer (ROC-score, 0.9107; $\mathrm{p}<0.001$ ) and RA and mammary cancer (ROC-score, $1 ; \mathrm{p}<0.001$ ).

\section{CONCLUSIONS}

Here, we provide the first experimental evidence showing that the presence of a growing tumor at a distant site induces specific changes in brain gene expression. The gene expression profiles were specific of each cancer type and differed from that induced by rheumatoid arthritis suggesting that different chronic pathologies can elicit a different gene expression profile in discrete brain regions.

It has been postulated for years the existence of an intricate network by which the nervous system can interact with peripheral systems. The CNS establish anatomical and soluble communications with organs such as the liver, spleen, gastrointestinal tract and others to allow the acquisition of peripheral information to be integrated and analyzed. Moreover, the CNS and the periphery exhibit reciprocal expression of receptors and ligands not only in the nervous and immune cells but also in the endocrine cells as well, suggesting that a bifunctional communication exists. Despite the fact that only a low percentage of differentially expressed genes were shared by the different models, the three models induced the largest changes in the hypothalamus confirming this region as the most relevant brain region involved in integrating peripheral information [6]. In this regard, a wide change in transcript levels associated with immune response was highlighted in the hypothalamic region of the colon cancer model as a late response at 8 days when the tumor is clearly noticeable. The question as to whether this response is specific of this tumor model because of its high intrinsic immunogenicity remains open but might suggest that antitumor immune responses might appear late in tumor growth.

Functional analysis of the genes validated by RT-PCR highlighted a number of genes involved in synaptic transmission that showed down regulated mRNA expression. We observed down-regulation of Gabarapl2, a protein involved in vesicle transport and axonal elongation in mammalian neurons [26] and ataxin-2, a protein associated with spinocerebellar ataxia type 2 ; it is of note that a subset of patients with parkinsonian phenotype responded to dopamine treatment suggesting a link between ataxin- 2 and dopamine [39]. On the other hand, previous studies have shown both decreased dopaminergic activity and increase in DOPAC:DA ratio in the hypothalamus of mice injected with mammary tumor cells and lymphoma cells, respectively [11, 40]. Moreover, administration of L-deprenyl that prevents breakdown of dopamine, inhibited mammary tumor growth suggesting that tumor development might be associated with decreased dopamine signaling [40]. In fact, our own data indicated a decrease in dopamine levels in specific brain 
A

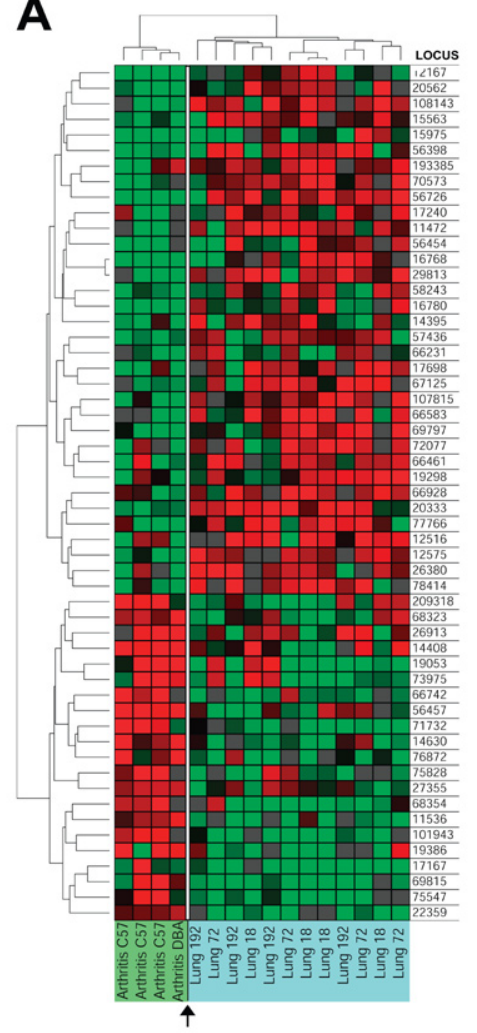

B

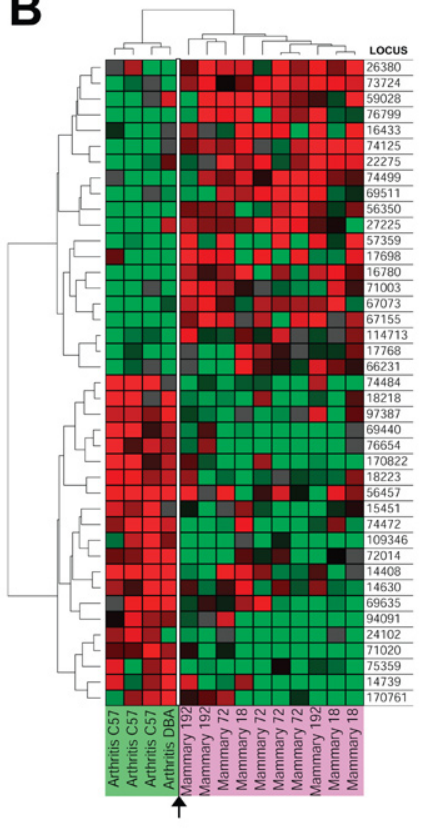

C
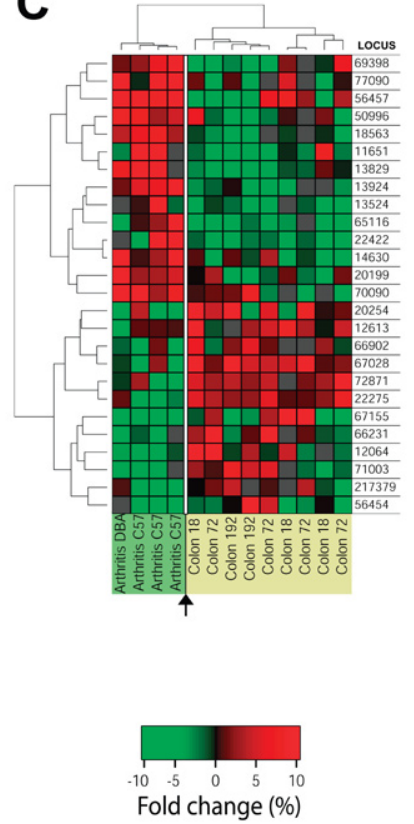

Fig. (5). Hierarchical cluster analysis of paired RA and cancer model. Hierarchical cluster analysis of hypothalamic samples from arthritis and each of the different cancer models, based on the subset of genes responding differentially between the different paired models. RA samples were labelled with green. For more details see Fig. (3).

areas in the presence of a tumor at a distant site (Casala et al. to be submitted) suggesting a potential link between the presence of a distal tumor and changes in neurotransmitter levels.

Peripheral production of inflammatory cytokines reprioritize the behavior of the animal, a process commonly known as sickness behavior [41] Several differentially expressed genes observed in the microarray study led us to assess whether genes involved in the "sickness behavior" process changed their expression levels. One feature of the sickness behavior response is diminished appetite. It was of interest that Oxt was one of the unique genes associated with sickness behavior that showed increased mRNA levels in the hypothalamus of mice injected with lung cancer cells. Oxt is referred as to a "satiety hormone" and it is known to induce reduction in food intake [42]. It was also interesting to find decreased Indo levels in all tumors. Lower levels of this transcript suggest greater accessibility of tryptophan to produce serotonin, a neurotransmitter associated with cancer related-cachexia, a process characterized by reduced food intake [37, 43]. Decreased expression levels of $\mathrm{Npy}, \mathrm{Mc} 4 r$ and $M c 5 r$ might also precede altered feeding behavior and response to stress that occurs later in cancer development [44]. Thus, the whole data indicate the generation of a molecular environment prone to the development of a behavioral response to sickness including reduced food intake, suggesting that the brain could modulate the expression levels of certain genes long before behavioral changes are manifested or that the tumor mass is noticeable. Recent studies indicate that anorectic rats bearing prostate adenocarcinoma showed increased IL-1 and IL-1Ra levels in the hypothalamus and other discrete brain regions [45]. However, no difference was observed in the expression levels of NPY or POMC mRNA at the later time points when rats became anorectic. Thus, it can be suggested that changes in the levels of molecules involved in food intake such as NPY occur early before anorexia is noticeable, while IL-1 increase associated to anorexia/cachexia occurs at a later point [45].

A distinctive gene profile was observed in each tumor type that was region-specific and differed from that observed in two established RA models. Although we cannot rule out that additional organs might exhibit changes in gene expression own to the presence of distal progressing tumors, the liver showed no specific expression indicating that the brain might be one of the earliest organs to show specific molecular changes in the presence of distant tumors. An intriguing possibility is that the sets of genes identified in this study play not only a role in disease recognition but also as part of a bidirectional and functional communication between the CNS and a peripheral disease. This might have important clinical implications in the future, especially if gene expression levels in the brain can be detected [46] and eventually pharmacologically regulated [47].

\section{CONFLICT OF INTEREST}

MCS is employee of Gentron Ltd. MR, FJP and OLP are members of the research career of CONICET and scientific advisors of Gentron Ltd 


\section{ACKNOWLEDGEMENTS}

We thank Cintia Serra and Daniela Celi for their excellent technical assistance and Guillermo Vilá Ortiz and Alejandra Ramirez for their participation at the early stages of the studies. We especially thank Yuti Chernajovsky and Adriana del Rey for careful reading of the manuscript and for their useful comments. This study was supported by Gentron Inc.

\section{SUPPLEMENTARY MATERIAL}

Supplementary material can be viewed at www.bentham.org/open/toneurj

\section{REFERENCES}

[1] Dunn GP, Bruce AT, Ikeda H, Old LJ, Schreiber RD. Cancer immunoediting: from immunosurveillance to tumor escape. Nat Immunol 2002; 3(11): 991-8.

[2] Khong HT, Restifo NP. Natural selection of tumor variants in the generation of "tumor escape" phenotypes. Nat Immunol 2002; 3(11): 999-1005.

[3] Ader R, Cohen N, Felten D. Psychoneuroimmunology: interactions between the nervous system and the immune system. Lancet 1995; 345(8942): 99-103.

[4] Besedovsky HO, del Rey A. Immune-neuro-endocrine interactions: facts and hypotheses. Endocr Rev 1996; 17(1): 64-102.

[5] Blalock JE. The immune system as the sixth sense. J Intern Med 2005; 257(2): 126-38.

[6] Steinman L. Elaborate interactions between the immune and nervous systems. Nat Immunol 2004; 5(6): 575-81.

[7] Tracey KJ. The inflammatory reflex. Nature 2002; 420(6917): 8539.

[8] Besedovsky H, del Rey A, Sorkin E, et al. The immune response evokes changes in brain noradrenergic neurons. Science 1983; 221(4610): 564-6.

[9] Turnbull AV, Rivier CL. Regulation of the hypothalamic-pituitaryadrenal axis by cytokines: actions and mechanisms of action. Physiol Rev 1999; 79(1): 1-71.

[10] Sternberg EM. Neural-immune interactions in health and disease. J Clin Invest 1997; 100(11): 2641-7.

[11] Chuluyan HE, Wolcott RM, Chervenak R, Dunn AJ. Catecholamine, indoleamine and corticosteroid responses in mice bearing tumors. Neuroimmunomodulation 2000; 8(3): 107-13.

[12] Deacon RM, Raley JM, Perry VH, Rawlins JN. Burrowing into prion disease. Neuroreport 2001; 12(9): 2053-7.

[13] Petit-Demouliere B, Chenu F, Bourin M. Forced swimming test in mice: a review of antidepressant activity. Psychopharmacology (Berl) 2005; 177(3): 245-55.

[14] Alvarez MJ, Vila-Ortiz GJ, Salibe MC, Podhajcer OL, Pitossi FJ. Model based analysis of real-time PCR data from DNA binding dye protocols. BMC Bioinform 2007; 8(1): 85

[15] Tran PH, Peiffer DA, Shin Y, et al. Microarray optimizations: increasing spot accuracy and automated identification of true microarray signals. Nucleic Acids Res 2002; 30(12): e54.

[16] Smyth GK, Speed T. Normalization of cDNA microarray data. Methods 2003; 31(4): 265-73.

[17] Pavlidis P. Using ANOVA for gene selection from microarray studies of the nervous system. Methods 2003; 31(4): 282-9.

[18] Pavlidis P, Noble WS. Analysis of strain and regional variation in gene expression in mouse brain. Genome Biol 2001; 2(10): 42.

[19] Ashburner M, Ball CA, Blake JA, et al. Gene ontology: tool for the unification of biology. The Gene Ontology Consortium. Nat Genet 2000; 25(1): 25-9.

[20] Hartigan JA. Clustering Algorithms. New York: John Wiley \& Sons Inc 1975.

[21] Schindler H, Wiese A, Auer J, Burtscher H. cRNA target preparation for microarrays: comparison of gene expression profiles generated with different amplification procedures. Anal Biochem 2005; 344(1): 92-101.
Harada M, Matsuzaki G, Shinomiya Y, et al. Generation of tumorspecific cytotoxic $\mathrm{T}$ lymphocytes in vivo by combined treatment with inactivated tumor cells and recombinant interleukin-2. Cancer Immunol Immunother 1994; 38(5): 332-8.

[23] Cirelli C, Gutierrez CM, Tononi G. Extensive and divergent effects of sleep and wakefulness on brain gene expression. Neuron 2004; 41(1): 35-43.

[24] Boesch SM, Donnemiller E, Muller J, et al. Abnormalities of dopaminergic neurotransmission in SCA2: a combined 123I-betaCIT and 123I-IBZM SPECT study. Mov Disord 2004; 19(11): 1320-5.

[25] Wullner U, Reimold M, Abele M, et al. Dopamine transporter positron emission tomography in spinocerebellar ataxias type 1,2, 3, and 6. Arch Neurol 2005; 62(8): 1280-5.

[26] Okazaki N, Yan J, Yuasa S, et al. Interaction of the Unc-51-like kinase and microtubule-associated protein light chain 3 related proteins in the brain: possible role of vesicular transport in axonal elongation. Brain Res Mol Brain Res 2000; 85(1-2): 1-12.

[27] Charron F, Tessier-Lavigne M. Novel brain wiring functions for classical morphogens: a role as graded positional cues in axon guidance. Development 2005; 132(10): 2251-62.

[28] Ramamurthy S, Mir F, Gould RM, Le Breton GC. Characterization of thromboxane A2 receptor signaling in developing rat oligodendrocytes: nuclear receptor localization and stimulation of myelin basic protein expression. J Neurosci Res 2006; 84(7): 1402-14.

[29] Furley AJ, Morton SB, Manalo D, et al. The axonal glycoprotein TAG-1 is an immunoglobulin superfamily member with neurite outgrowth-promoting activity. Cell 1990; 61(1): 157-70.

[30] Theodosis DT, Piet R, Poulain DA, Oliet SH. Neuronal, glial and synaptic remodeling in the adult hypothalamus: functional consequences and role of cell surface and extracellular matrix adhesion molecules. Neurochem Int 2004; 45(4): 491-501.

[31] Dunnick J, Blackshear P, Kissling G, et al. Critical pathways in heart function: bis(2-chloroethoxy)methane-induced heart gene transcript change in F344 rats. Toxicol Pathol 2006; 34(4): 348-56.

[32] Cheng JD, Valianou M, Canutescu AA, et al. Abrogation of fibroblast activation protein enzymatic activity attenuates tumor growth Mol Cancer Ther 2005; 4(3): 351-60.

[33] Tissier A, Kannouche P, Mauffrey P, et al. Molecular cloning and characterization of the mouse Kin17 gene coding for a $\mathrm{Zn}$-finger protein that preferentially recognizes bent DNA. Genomics 1996; 38(2): 238-42.

[34] Haque F, Lloyd DJ, Smallwood DT, et al. SUN1 interacts with nuclear lamin A and cytoplasmic nesprins to provide a physical connection between the nuclear lamina and the cytoskeleton. Mol Cell Biol 2006; 26(10): 3738-51.

[35] Mailloux RJ, Singh R, Appanna VD. In-gel activity staining of oxidized nicotinamide adenine dinucleotide kinase by blue native polyacrylamide gel electrophoresis. Anal Biochem 2006; 359(2): 210-5.

[36] Wurmbach E, Yuen T, Sealfon SC. Focused microarray analysis Methods 2003; 31(4): 306-16.

[37] Konsman JP, Parnet P, Dantzer R. Cytokine-induced sickness behaviour: mechanisms and implications. Trends Neurosci 2002; 25(3): 154-9.

[38] Guenther K, Deacon RM, Perry VH, Rawlins JN. Early behavioural changes in scrapie-affected mice and the influence of dapsone. Eur J Neurosci 2001; 14(2): 401-9.

[39] Lu CS, Wu CYH, Yen TC, et al. Dopa-responsive parkinsonism phenotype of spinocerebellar ataxia type 2. Mov Disord 2002; 17(5): 1046-51.

[40] ThyagaRajan S, Madden KS, Stevens SY, Felten DL. Anti-tumor effect of L-deprenyl is associated with enhanced central and peripheral neurotransmission and immune reactivity in rats with carcinogen-induced mammary tumors. J Neuroimmunol 2000; 109(2): 95-104.

[41] Dantzer R. Cytokine-induced sickness behaviour: a neuroimmune response to activation of innate immunity. Eur J Pharmacol 2004; 500(1-3): 399-411

[42] Wesolowski SR, Allan MF, Nielsen MK, Pomp D. Evaluation of hypothalamic gene expression in mice divergently selected for heat loss. Physiol Genomics 2003; 13(2): 129-37. 
[43] Laviano A, Russo M, Freda F, Rossi-Fanelli F. Neurochemical mechanisms for cancer anorexia. Nutrition 2002; 18(1): 100-5.

[44] Heilig M, Thorsell A. Brain neuropeptide Y (NPY) in stress and alcohol dependence. Rev Neurosci 2002; 13(1): 85-94.

[45] Plata-Salaman CR, Wilson CD, Ffrench-Mullen JM. In vivo IL1beta-induced modulation of G-protein alphaO subunit subclass in the hypothalamic ventromedial nucleus: implications to IL-1beta- associated anorexia. Brain Res Mol Brain Res 1998; 58(1-2): 18894.

[46] Liu CH, Huang S, Cui J, et al. MR contrast probes that trace gene transcripts for cerebral ischemia in live animals. FASEB J 2007; 21(11): 3004-15.

[47] Gidron Y, Perry H, Glennie M. Does the vagus nerve inform the brain about preclinical tumours and modulate them? Lancet Oncol 2005; 6(4): 245-8.

(C) Alvarez et al.; Licensee Bentham Open.

This is an open access article licensed under the terms of the Creative Commons Attribution Non-Commercial License (http://creativecommons.org/licenses/by-nc/3.0/) which permits unrestricted, non-commercial use, distribution and reproduction in any medium, provided the work is properly cited. 